\title{
Research Paper \\ Comparison of Resilience and Self-Efficacy in Athletes and Non-Athletes With Disabilities Caused by Spinal Cord Injury
}

\author{
Niloufar Dirmanchi ${ }^{1}{ }^{(0,}{ }^{*}$ Mohammad Saeed Khanjani $^{2}$ (C)
}

1. MSc. Student of Rehabilitation Counseling, Department of Counseling, Faculty of Psychology and Educational Sciences, South Tehran Branch, Islamic Azad University Tehran, Iran.

2. PhD. in Rehabilitation Counseling, Department of Rehabilitation Management, University of Social Welfare and Rehabilitation Sciences, Tehran, Iran.

\begin{tabular}{|c|c|}
\hline $\begin{array}{l}\text { Use your device toscan } \\
\text { and read the article online }\end{array}$ & Cftation Dirmanchi N, Khanjani MS. Comparison of Resilience and Self-Efficacy in Athletes and Non-Athletes With Dis- \\
\hline 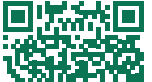 & $\begin{array}{l}\text { abilities Caused by Spinal Cord Injury.Iranian Journal of Psychiatry and Clinical Psychology. 2019; 25(2):150-163. http://dx.doi. } \\
\text { org/10.32598/ijpcp.25.2.150 }\end{array}$ \\
\hline a phist & doi"http://dx.doi.org/10.32598/ijpcp.25.2.150 \\
\hline
\end{tabular}

Received: 21 Dec 2018

Accepted: 05 Feb 2019

Available Online: $01 \mathrm{Jul} 2019$

Key words:

Resilience, Self-efficacy,

Spinal Cord Injury,

Disabled

\begin{abstract}
A B S TRACT
Objectives The current causal-comparative study aimed at comparing resiliency and self-efficacy between athletes and non-athletes with disabilities caused by Spinal Cord Injury (SCI).

Methods Using simple random sampling method, 32 subjects were enrolled in each group. Data were collected using the Connor-Davidson Resiliency Scale and the Sherer general self-efficacy questionnaire. Results The findings of the study showed that athletes with disabilities had more resilience than their non-athlete counterparts $(P=0.006)$. However, considering the score of 50 as the appropriate resiliency threshold, there was no significant difference in resilience between the two groups, and the total selfefficacy score, with mode of 65 , was higher in the athletes than non-athletes ( $P=0.001)$. Also, the findings of the study showed a significant relationship between resilience and self-efficacy, and doing exercise. And sports can be suggested as a way to promote resilience and self-efficacy in people with SCl.

Conclusion According to the findings of the present study, there is a significant relationship between resilience and self-efficacy, and doing exercise. Exercise is likely to be a way to promote resilience and self-efficacy in people with $\mathrm{SCl}$.
\end{abstract}

\section{Extended Abstract}

\section{Introduction}

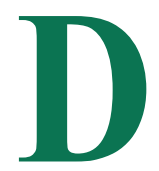

isability is a general term refers to the condition that restricts activities and prevents people from participation in activities [1]. The United Nations Convention on the Rights of Persons with Disabilities in 2007 stated that people with disabilities include those with a long-term physical, psychological, and intellectual disabilities that, in the face of various barriers, may not be fully and effectively interact with others in the society [2].
Spinal Cord Injury (SCI) is one of the commonest types of disability. The spinal cord is a super-central part of the nervous system, and even a small damage to it can lead to severe disability [3]. Garmezy and Masten defined the resilience as "the process or the consequence of successful adaptation in spite of challenging and threatening conditions" [10]. Also, self-efficacy is a concept derived from the social cognitive theory of Albert Bandura [13]. Feeling self-efficacy enables individuals to work hard by using skills to deal with obstacles. Self-efficacy is related to health outcomes and is an indicator of post-traumatic injury rehabilitation.

-

* Corresponding Author:

Mohammad Saeed Khanjani, PhD.

Address: Department of Rehabilitation Management, University of Social Welfare and Rehabilitation Sciences, Tehran, Iran.

Tel: +98 (21) 22180061

E-mail: sa.khanjani@uswr.ac.ir 
Meanwhile, professionals recommend doing exercise as an effective treatment because of positive effects of sports activities on mental health of people with physical disabilities [7]. Many studies showed a significant difference between the resilience and self-efficacy of healthy subjects including athletes and non-athletes $[15,16,17]$. Therefore, the need for doing exercise in individuals with disabilities is more than others. Today, exercise is a major part of the activities recommended by the SCI centers worldwide. The current study question thereby was that whether exercise can have a positive effect on the resilience and self-efficacy of individuals with disabilities caused by SCI.

\section{Method}

The current causal-comparative study was conducted on athletes with spinal cord injury referring to the National Paralympic Academy as well as non-athlete patients who developed disabilities following SCI referring to the Protection Centre of Spinal Cord Disables of Iran. The instruments used in the research were the Conor-Davidson Resilience Scale (CD-RIS) and the general self-efficacy questionnaire developed by Sherer et al. The subjects were selected using simple ransom sampling method. Of the 60 members of the National Paralympic Academy and 200 members of the Protection Centre of Spinal Cord Disables of Iran, 32 subjects who met the inclusion criteria were randomly selected for each group. The sample size with the assumption of the first and second type errors 5\% and 20\% respectively, and the mean and standard deviation of self-efficacy in athletes and non-athletes were 53.62, 15.59, 12.65, And colleagues [17], and based on the comparison formula, the mean of Stata software was calculated to be 32 in both groups.
Descriptive statistics used in the study were mean, standard deviation, percentage, mean and mean quark distortion; inferential statistics included independent t-test and the Mann-Whitney U test. In addition, Chi-square test was used to compare resilience and self-efficacy between athlete and non-athlete groups, and Pearson correlation coefficient was used to examine the relationship between the duration of disability with resilience and self-efficacy.

\section{Results}

Based on the results of $\mathrm{t}$ - and the Mann-Whitney $\mathrm{U}$ tests, there was no significant difference between the two groups in terms of age, gender, educational level, marital status, and stage of SCI. Meanwhile, the mean duration of disability had significant difference between the groups $(\mathrm{P}=0.001)$.

The mean of resiliency in athletes and non-athletes was 72 and 63, respectively, showing a significant difference between the two groups $(\mathrm{P}=0.006)$. Also, there was a significant difference in self-efficacy between athletes and non-athletes $(\mathrm{P}=0.001)$ (Table 1$)$; in other words, athletes with SCI had a better self-efficacy than their non-athlete counterparts. The study findings showed a positive correlation between self-efficacy and resilience, and correlation coefficient of 0.549 (Table 2).

\section{Discussion}

Regarding the findings of the present study and considering the results of previous studies, it can be concluded that SCI causes serious complications in all personal aspects, including physical, psychological, and social health, and may be associated with higher resilience and self-efficacy.

Table 1. Comparison of resilience and self-efficacy between athletes and non-athletes with disabilities caused by SCI

\begin{tabular}{cccccccc}
\hline \multirow{2}{*}{ Item } & Athlete & Non-Athlete & P & \multicolumn{3}{c}{ Athlete/Non-Athlete } \\
\cline { 5 - 8 } & & & & \multicolumn{2}{c}{ Minimum } & Maximum \\
\hline Resilience & $72.4 \pm 12.5$ & $63.0 \pm 13.6$ & 0.006 & 43 & 34 & 97 & 99 \\
Self-efficacy & $69.5 \pm 12.5$ & $62.0 \pm 11.5$ & 0.001 & 46 & 36 & 82 & 76 \\
\hline
\end{tabular}

Table 2. Correlation of resilience and self-efficacy in athletes and non-athletes with SCI

\begin{tabular}{ccc}
\hline & \multicolumn{2}{c}{ Self-Efficacy } \\
\cline { 2 - 3 } Item & Pearson Correlation Coefficient & $\mathbf{P}$ \\
\hline Resilience & 0.549 & 0.001 \\
\hline
\end{tabular}


According to the findings of the study, there is a significant relationship between resilience and self-efficacy, and doing exercise. Doing exercise is likely to be a way to promote resilience and self-efficacy in people with SCI.

Studies showed the positive effects of physical activity, exercise, or sports on physical and mental health of people with physical disabilities; i. e. better mood management and reduced stress, depression, and anxiety [7]. Kornhaber et al. [14] also argued that the ultimate goal of rehabilitation in patients with SCI is to maximize self-efficacy. Continuous reinforcement is in fact the ability of the individual to build self-efficacy and resilience, which is important among the ones with disabilities. Therefore, self-efficacy and resilience are the major factors for the repercussions of patients with SCI. Therefore, according to the results of the current study, there is a high level of resilience and self-efficacy in the athletes with disabilities. It is also recommended to implement sport techniques in welfare centers and other organizations serving patients with SCI to improve their psychological functioning.

\section{Ethical Considerations}

Compliance with ethical guidelines

In this research all ethical considerations such as interview voluntariness, secrecy of information and informed consent were observed.

\section{Funding}

The paper was extracted from the Master thesis of the the first author at Faculty of Psychology and Educational Sciences, Islamic Azad University, South Tehran Branch, Iran.

\section{Authors' contribution}

Conceptualization, methodology, review and editing: All authors; Investigation, resources, data curation, original draft preparation, visualization: Niloufar Dirmanchi; Suprvision: Mohammad Saeed Khanjani.

\section{Conflict of interest}

The authors declared no conflict of interest.

\section{Acknowledgment}

The authors would like to thank Dr. Ali Kabir (Head of the Center for Education \& Development of Iran University of Medical Sciences), Dr. Hadizadeh (Statistical Consultant), Head of the Department of of Sports Psychology at Nation- al Olympic and Paralympic Academy, , Management and Personnel of the Center of Support For Spinal Cord Disabilities, and all participants for their valuable cooperation. 


\title{
مقايسه تابآورى و خودكار آمدى معلولين ضايعه نخاعى ورزشكار و غيرورزشكار
}

\author{
نيلوفر ديرمانجى '، "محمدسعيد خانجانى 'ه \\ ا - دائشوى كارشئاسي ارشد مشاوره توانبخشي، كروه مشاوره، دانشكده روائشناسي و علوم تربيتي، واحد تهران جنوب، دانشكاه آزاد اسلامي، تهران، ايران.

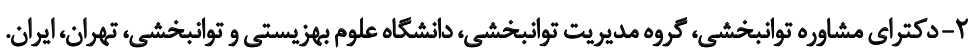

\section{حكبد}

هدافت هدف يروهش حاضر مقايسه ثابآورى و خودكارآمدى بوده است بين معلولين غيرورزشكار و معلولينى كه به صورث حرفهاي ورزش مى مكنئ مان

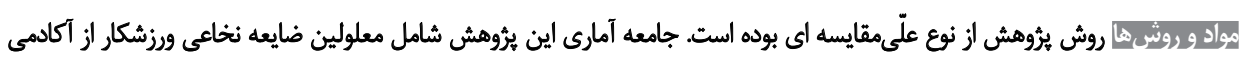

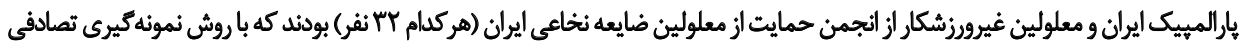

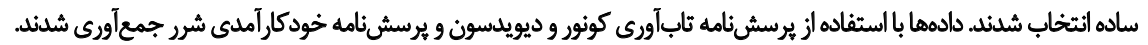

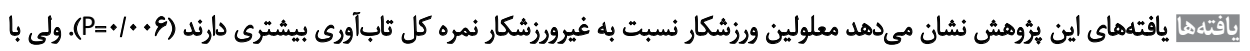

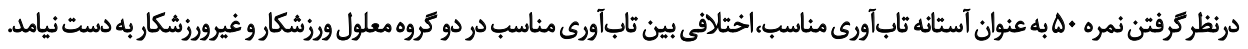

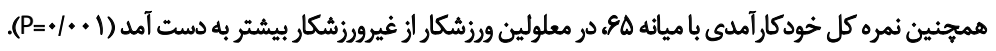

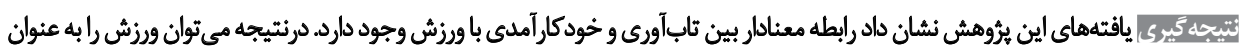

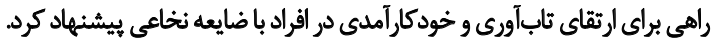

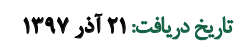

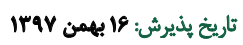

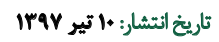

كليدوازوها:

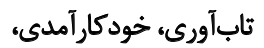
معلوليت ضايعه نخآئي، ناثوان

تخمين زده شده است [1]. از جمله معلوليتهاء آسيب طناب

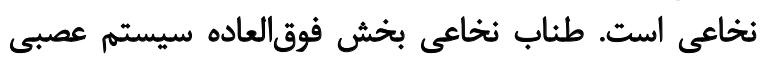

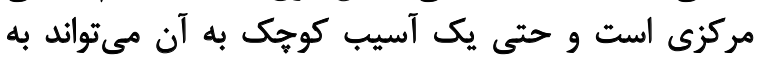

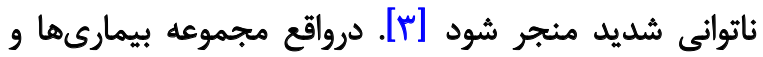

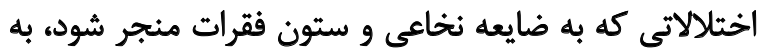

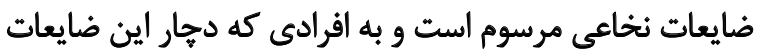

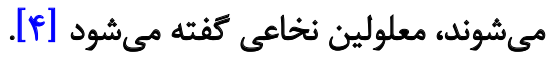

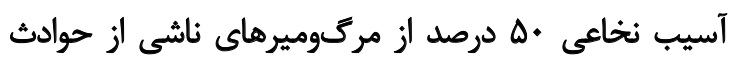

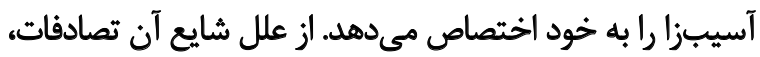

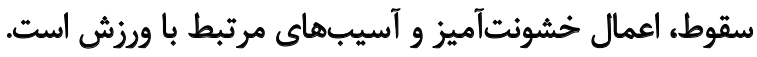

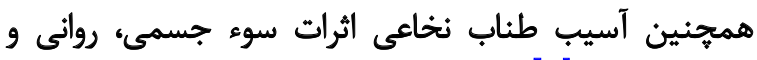

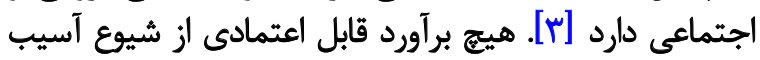

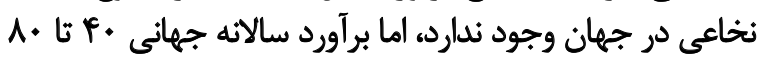

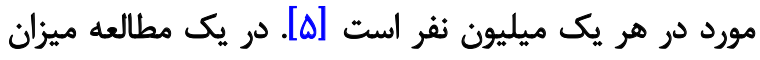

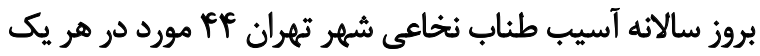

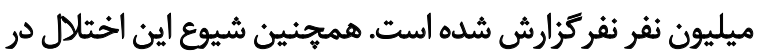

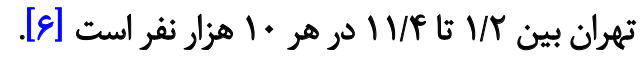

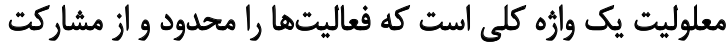

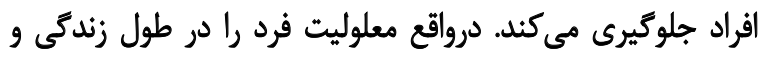

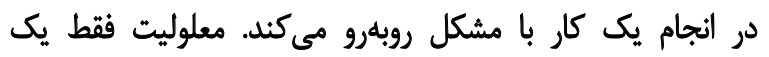

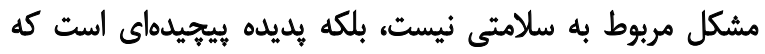

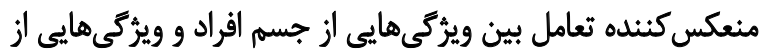

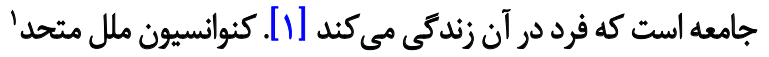

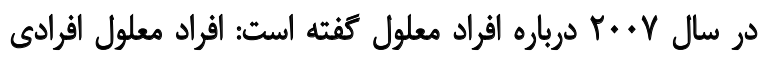

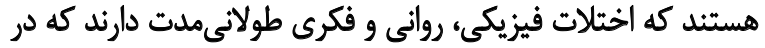

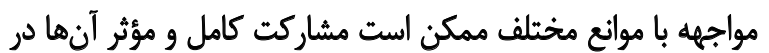

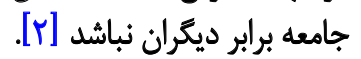

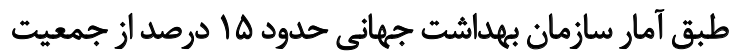

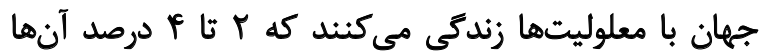

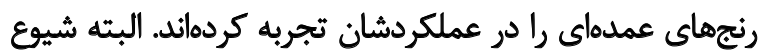

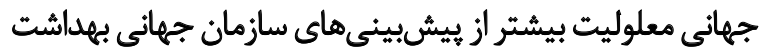

1. United Nations Convention

: تويسئده مسئول:

دكتر محمدسعيد خانجانى

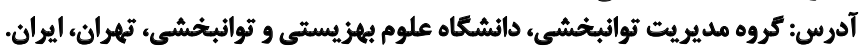

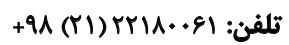
sa.khanjani@uswr.ac.ir يست الكترونيكي 


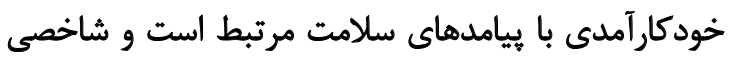

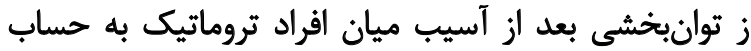

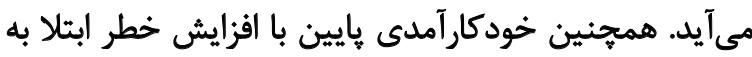

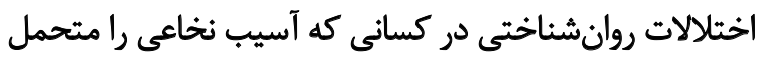

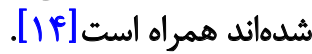

در اين ميان به عنوان بخشى از برنامه توانبخشى بيماران

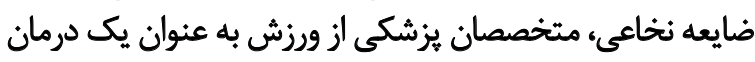

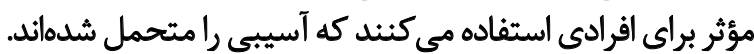

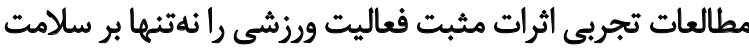

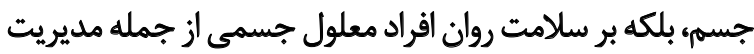

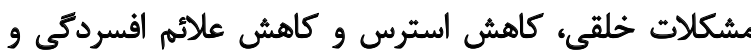
اضطراب نشان داده است كامث [V]

در بسيارى از يروهشهاى انجام

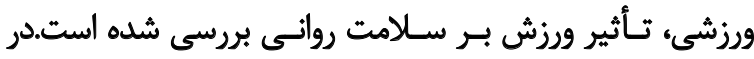

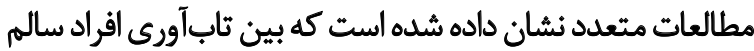

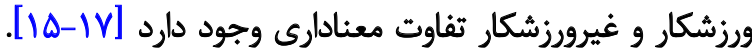

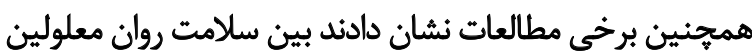

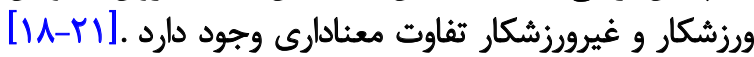

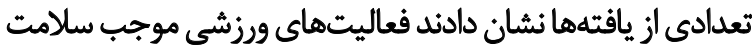

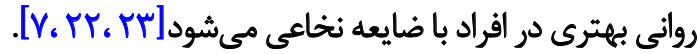

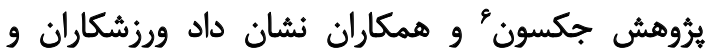

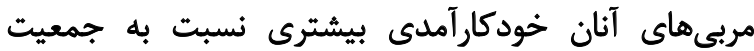

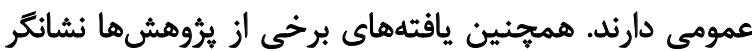

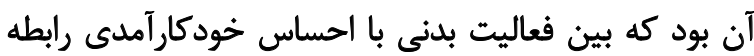

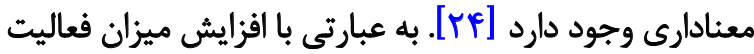

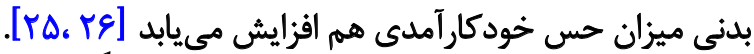

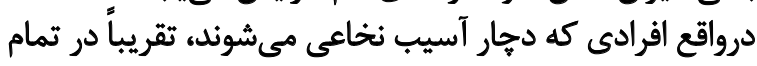

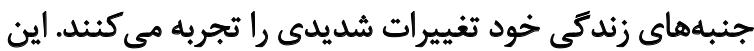

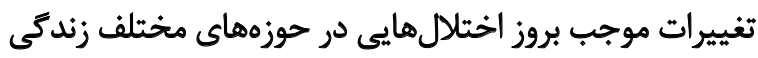
اعم از جسمى، روانى و اجتماعى مى شورد.

همجنين سازكارى مثبت با اين آسيب اغلب نياز به تغييرات

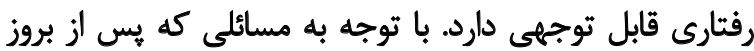

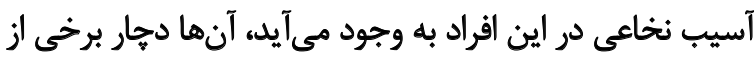

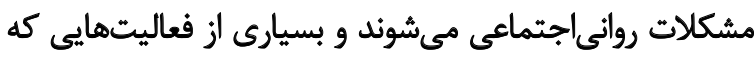

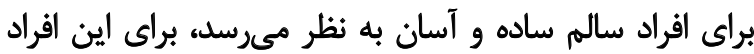

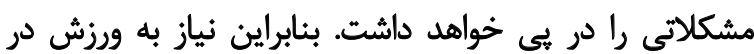

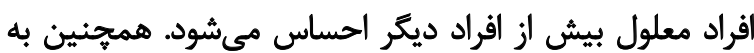

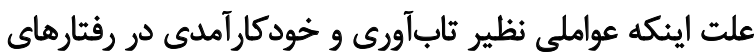

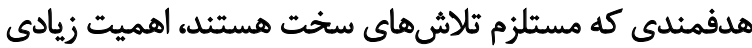

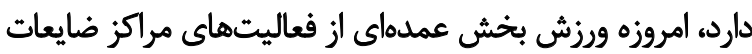
نخاعى در سراسر دنيا را به خود اختصاص عمداص مي دهاليتداي
محققان به طور كلى تابآورى را به عنوان يك سازكارى مثبت با باري

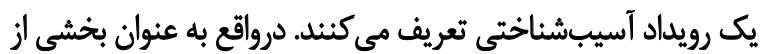

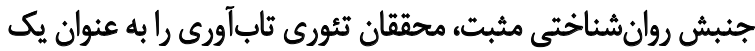

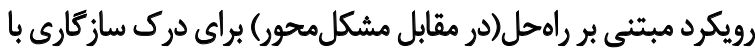

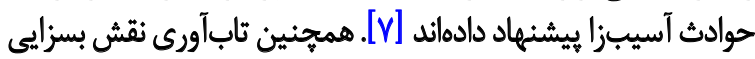

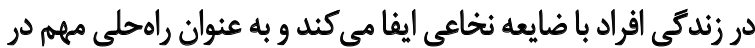

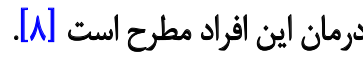

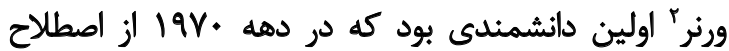

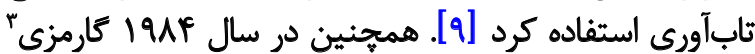

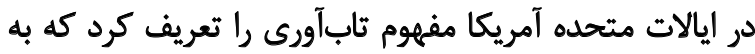

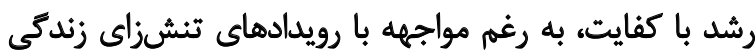

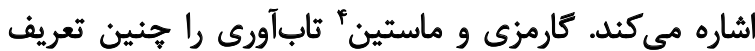

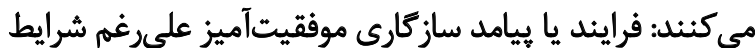

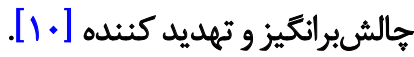

واره تابآورى شامل مفهوم انعطافيذيرى، بهبود و بازكشت إيت

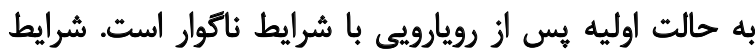

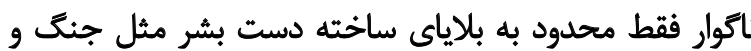

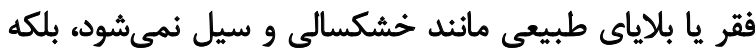

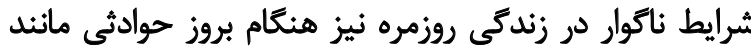

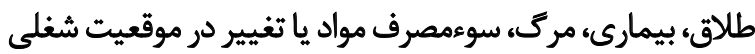

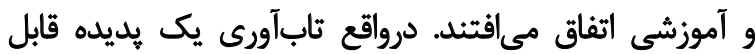

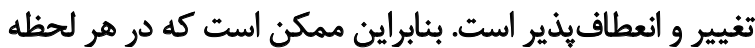

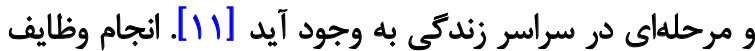

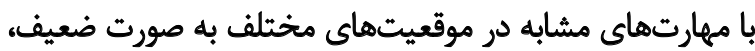

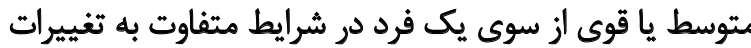

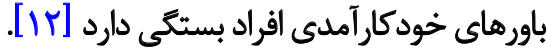
خودكارآمدى مفهومى بركرفته از نظريه شناختىاجتماعتى بندي

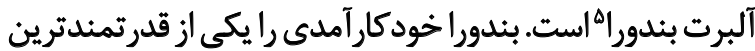

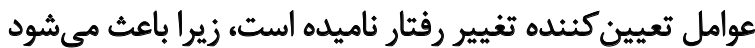

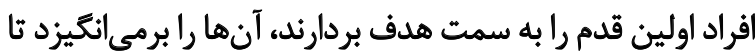

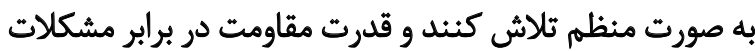

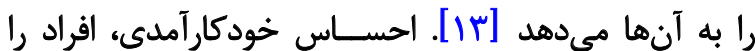

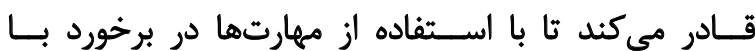

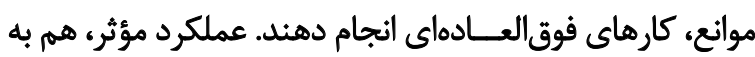

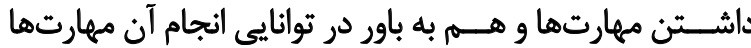

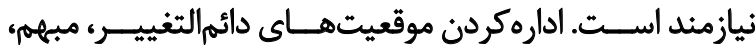
غيرقابل يُيشبينى و اســترسزا، مستلزم داشتن مهارتهاتي

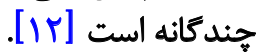

2. Werner

3. Garmezy

4. Masten

5. Bandura 
بورشش ثامه ثاب آورى كوثور و ديويدسون

براى سنجش تاب آورى، از مقياس ثابآورى كونور وديويدسون

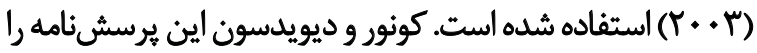

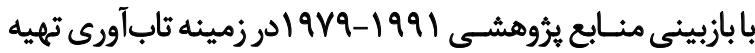

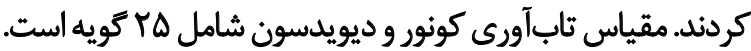

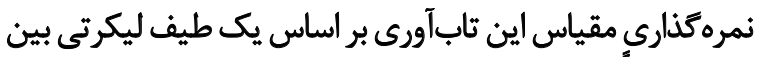
صفر (كاملاً نادرست) تا جهار (هميشه درست) است

در تفسير مقياس تابآورى هرجه فرد نمره بيشترى بكيرد،

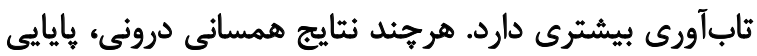

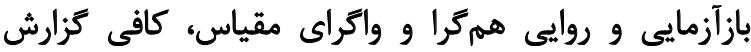

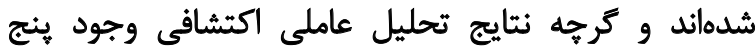
عامل (شايستىى لاستحكام شخصى، اعتماد به به غرايز شخصى

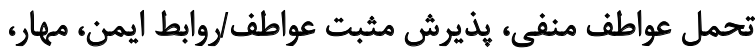

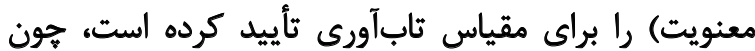

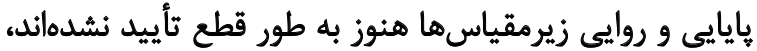

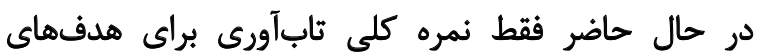

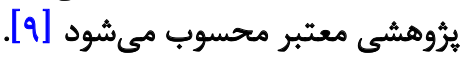

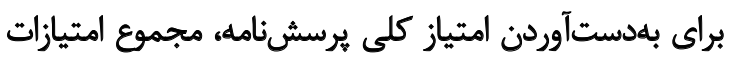

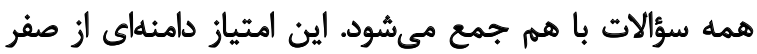

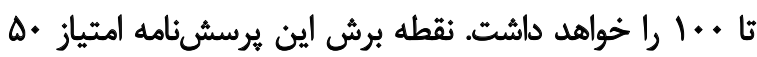

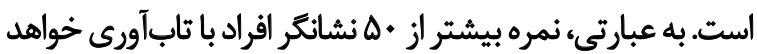

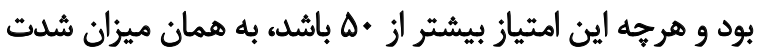

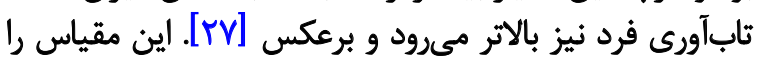

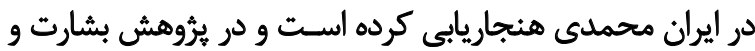

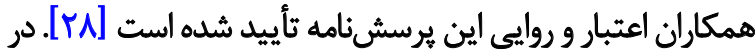

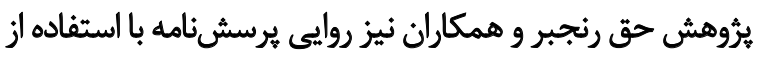

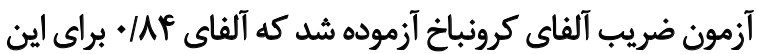

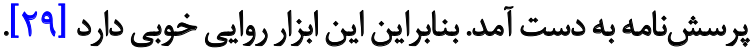

$$
\text { ير سشئامه خُود كار آمدى عمومى شرر. }
$$

مقياس خودكارآمدى عمومى را شرر و همكاران در سال

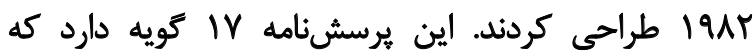

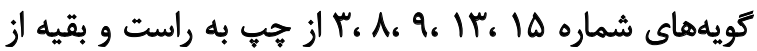

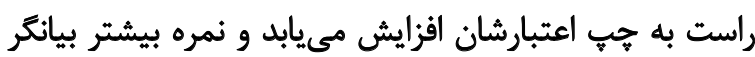

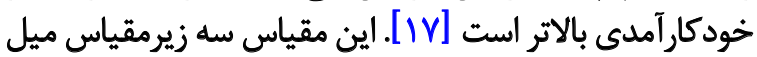

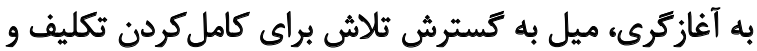

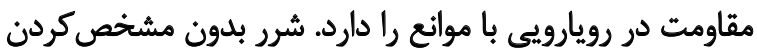

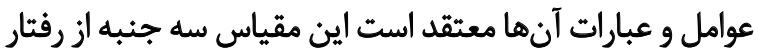

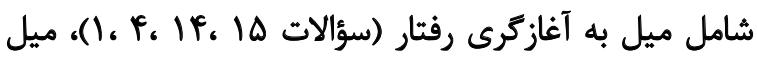

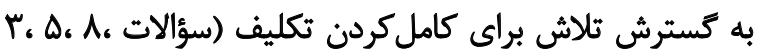

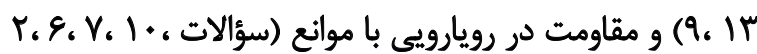
ا

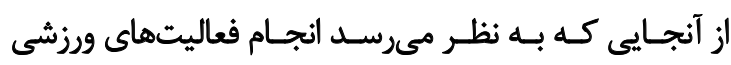

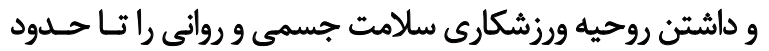

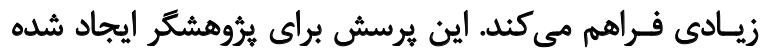

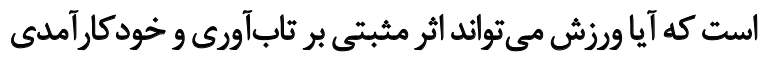

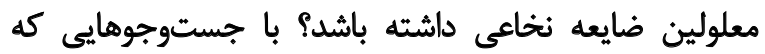

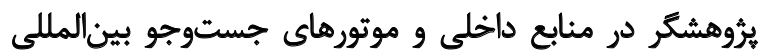

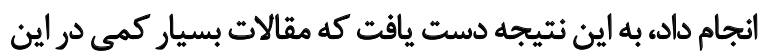

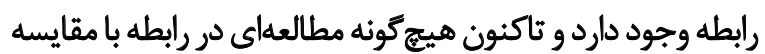

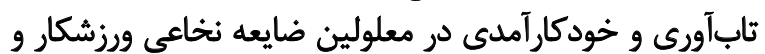

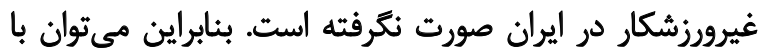

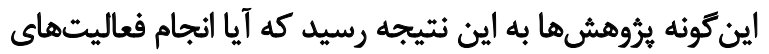

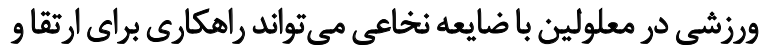
حفظ تابآورى و خودكار آمدى در اين افراد باشيد.

ورش

اين بروهش از نوع علّ مقايسهاى است. آزمودنى هاى ثيثروهش

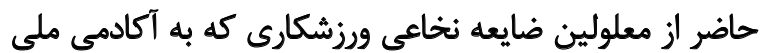

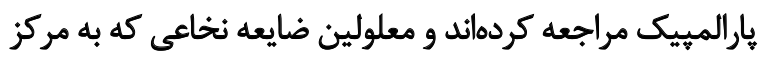

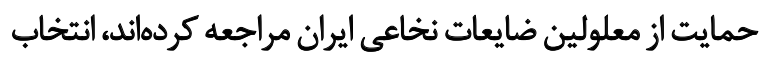

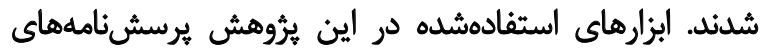

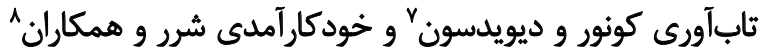
است. روش نمونه كيرى به صورت كمارش تصادفي ونى بوده است

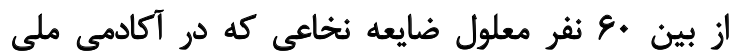

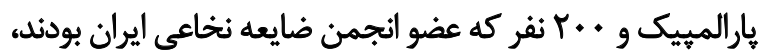

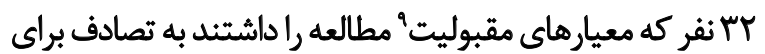

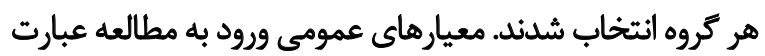

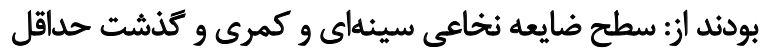

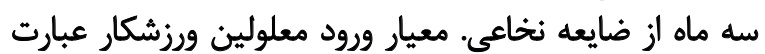

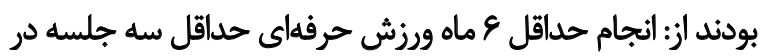

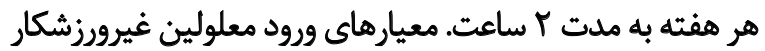

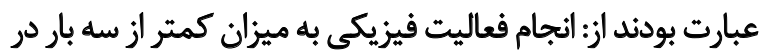

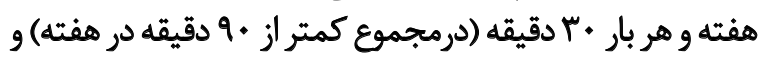

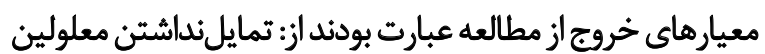
به يركردن يرسش نامه و داشتن ضايعه همراه ( هماند آسيبنهاي مغزى، فلج مغزى و تشنج كنترلنشده).

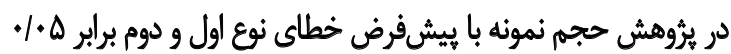

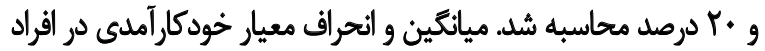

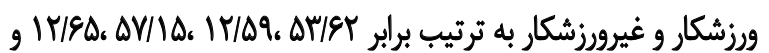

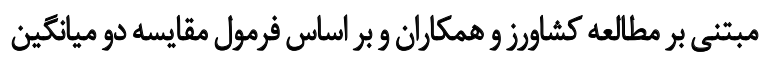

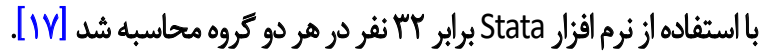

7. The Connor-Davidson resilience scale (CD-RISC)

8. General Sherer Self-efficacy Scale (GSES)

9. Eligibility 
خردهمقياس كنترل شخصى، مقياس درجه اجتماعى مارلو و ورئي

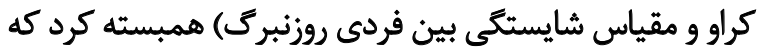

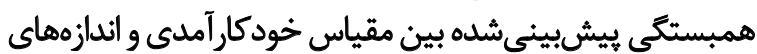

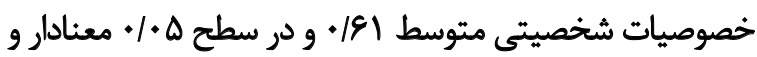
در جهت تأييد سازه مدنظر بود.

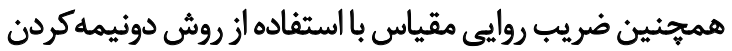

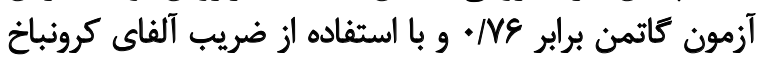

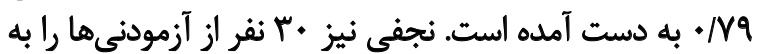

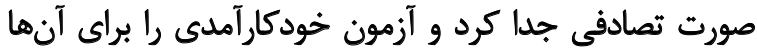

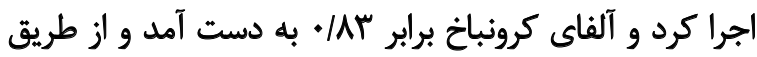

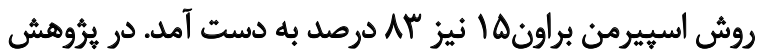

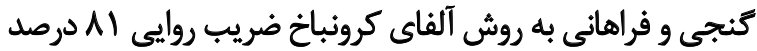

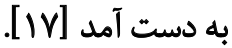

براى تحليل دادهها در بثروهش حاضر در بخش آمار توصيفى از

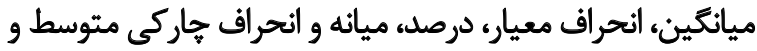

15. Spearmanbrown

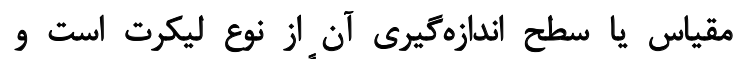

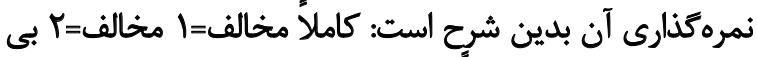

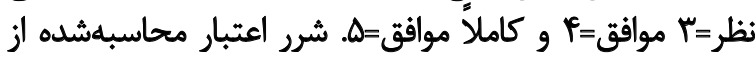

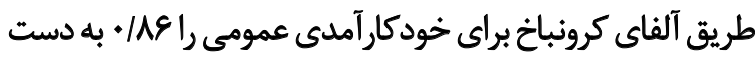

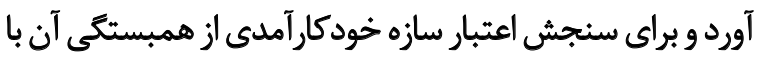

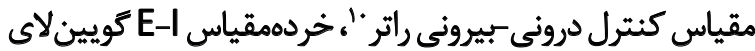

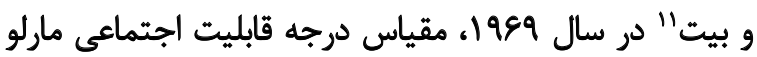

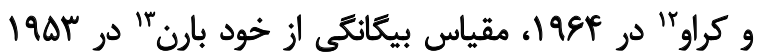

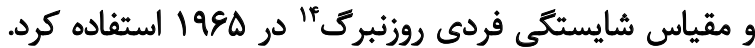

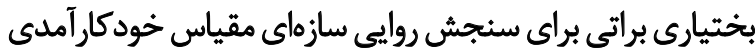

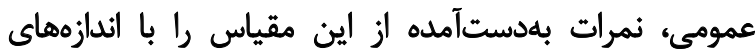
جندين ويثَّى شخصيتى (مقياس كنترل دورنى و بيرونى راتر،

10. Rotter

11. Gueinla and Bit

12. Marlow and crowne

13. Baron

14. Rosenberg

جدول ا. مقايسه ورزشكاران و غيروز شكاران از نظر وضعيت جمعيتشناختى

\begin{tabular}{|c|c|c|c|c|c|c|c|c|}
\hline \multicolumn{4}{|c|}{ ورزشكار/غيرورزشكار } & \multirow{3}{*}{ هعني سارى } & \multirow{3}{*}{ غيرورزشكار } & \multirow{3}{*}{ ورزشكار } & \multirow{2}{*}{\multicolumn{2}{|c|}{ مشخصات }} \\
\hline \multicolumn{2}{|c|}{ بيشترين } & \multicolumn{2}{|c|}{ كمترين } & & & & & \\
\hline \multirow[t]{11}{*}{ prof } & $\Delta r$ & M & iv & & & & \multicolumn{2}{|c|}{ 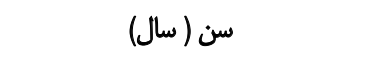 } \\
\hline & & & & ./pmif & $19(\Delta Q / 4)$ & rr(ENA) & 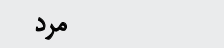 & \multirow{2}{*}{ جنس } \\
\hline & & & & - /4eq & $\operatorname{lr}(\varphi+18)$ & $1 \cdot(M / Y)$ & نj & \\
\hline & & & & & $1(\% / 1)$ & - & ابتدايي & \multirow{3}{*}{ تحيزان } \\
\hline & & & & & $M(\varepsilon \Delta / 8)$ & $W(\Delta \& / M)$ & راهنمايى و دييرستان & \\
\hline & & & &.$/ M V A$ & $1 \cdot(r / T)$ & $I f(F / A)$ & تحصيلات دانشكاهي & \\
\hline & & & & & $10(\% \& / 9)$ & $\operatorname{Ir}(\varphi+19)$ & 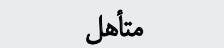 & \multirow{3}{*}{ تأهل } \\
\hline & & & &.$/ \Delta \Delta V$ & $\operatorname{IV}(\Delta r / 1)$ & $W(\Delta \& / T)$ & مجرد & \\
\hline & & & & & - & $I(Y / 1)$ & مطلقه & \\
\hline & & & & $\cdot / \Delta \Delta F$ & $r \cdot(q r / A)$ & $r(q \& / 9)$ & سينهاي & سطح \\
\hline & & & & & $r(\xi / r)$ & $1(r / 1)$ & 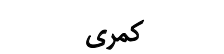 & 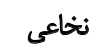 \\
\hline \multirow[t]{3}{*}{ mer } & PA. & $f$ & $\operatorname{mp}$ & $1 \cdots 1$ & 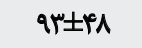 & $(M T \pm Y)$. & \multicolumn{2}{|c|}{ مدتزمان معلوليت (ماه) } \\
\hline & FA. & & $\pi$ & & & IrTIAf & \multicolumn{2}{|c|}{ ملت زمان ورزش حرفهاي (ماه) } \\
\hline & rra & & ir & & & $r q \pm r$ & \multicolumn{2}{|c|}{ مدتزمان عضويت در ثيهم ملى(ماه) } \\
\hline
\end{tabular}


جدول r. مقايسه تابآورى و خودكارآمدى در معلولين با ضايعه نخاعى ورزشكار و غيرورزشار

\begin{tabular}{|c|c|c|c|c|c|c|c|}
\hline \multicolumn{4}{|c|}{ وزظشكار/غيرورزشكار } & \multirow{3}{*}{ معني مارئ } & \multicolumn{2}{|c|}{ ميانكين } & \\
\hline \multicolumn{2}{|c|}{ بيشترين } & \multicolumn{2}{|c|}{ كمترين } & & غيرورزشكار & ورزشكار & \\
\hline 99 & 9V & mp & Pr & & $q \Psi / N \pm \cdot / 8$ & $V T / / r \pm F / \Delta$ & تابآورى \\
\hline Ve & Ar & e & te & $.1 .+1$ & ET/I土+/D & $g q / 1 Y \pm \Delta / \Delta$ & خودكار آمدى \\
\hline
\end{tabular}

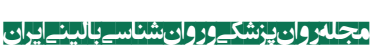

جدول r. رابطه تاب آورى با خودكارآمدى معلولين ورزشكار و غيرورزشكار

\begin{tabular}{|c|c|c|}
\hline \multicolumn{2}{|c|}{ خودكارآمدى } & \multirow{2}{*}{ متغير } \\
\hline سطح معنى دارى & ضريب همبستكى ييرسون & \\
\hline $.1 . .1$ & - lapq & تاب|آورى \\
\hline
\end{tabular}

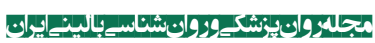

اين رابطه مطرح مىشود اين است كه آيا فعاليت ورزشى است

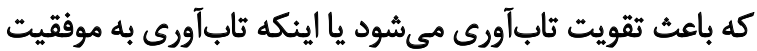

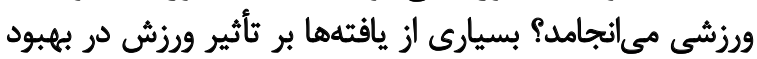

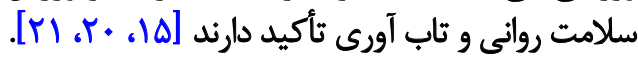

كشاورز و همكاران نيز نشان دادند ورزشكاران نظامى از نإن

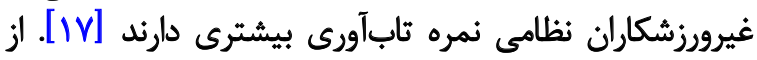

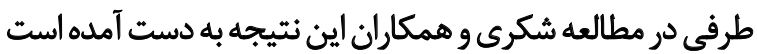

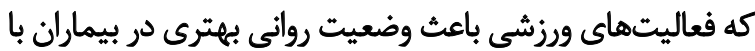

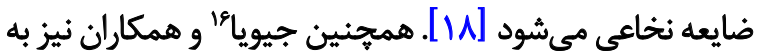

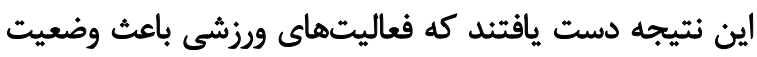

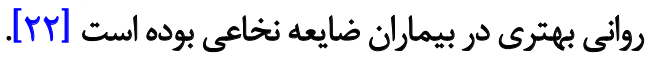
يافتههاي بخشايش و همكاران نيز نشان داد بين سلامت روان

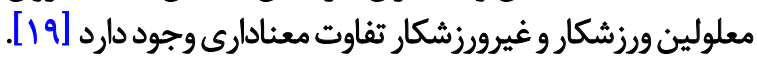

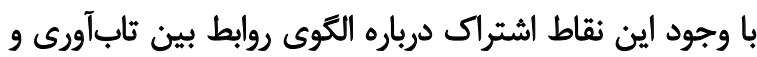

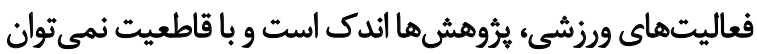

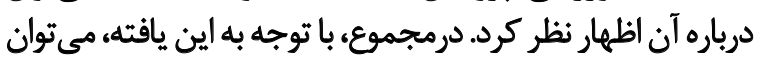

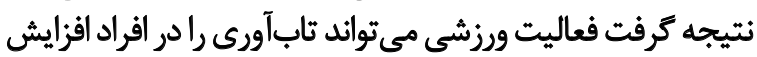

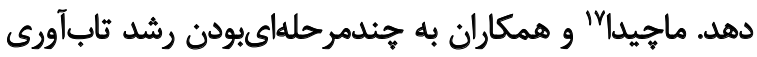

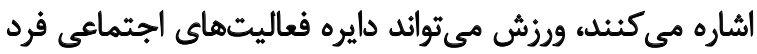

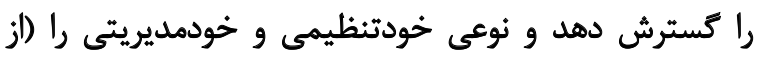

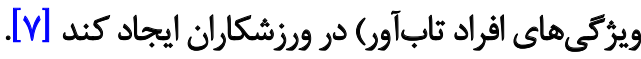

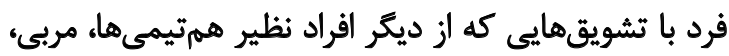

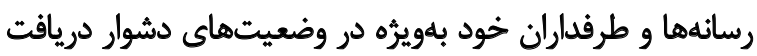

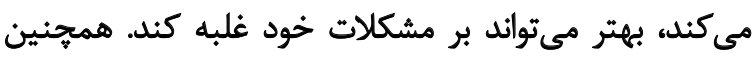
فعاليتهاى فيزيكى با شدت بالا (ضربان قلب بيش از •و ع درصد

16. Gioia

17. Machida

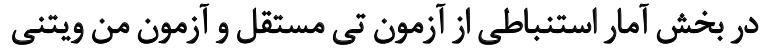

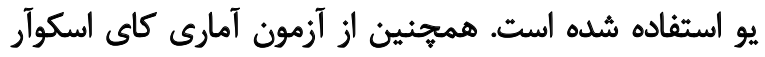

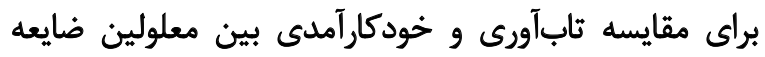

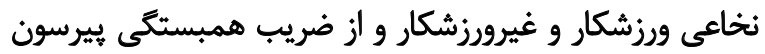

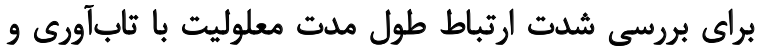
خود كارآمدى أستفاده شده است.

يافتهها همانطور كه در جدول شماره ا ملاحظه ميشود، در اين

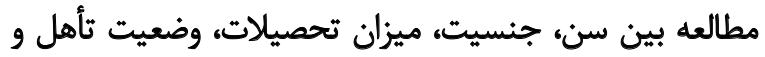

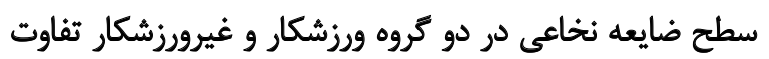

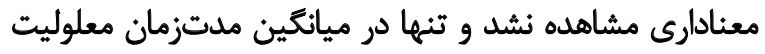
اختلاف معنادارى ديده شد.

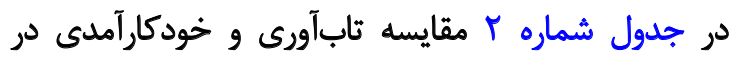

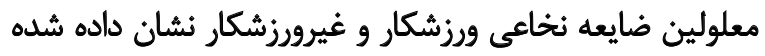

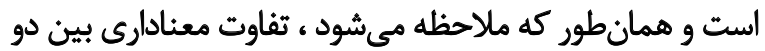

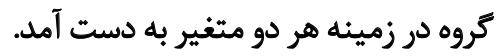

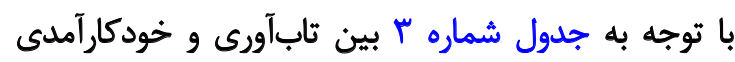

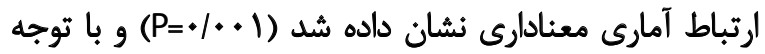

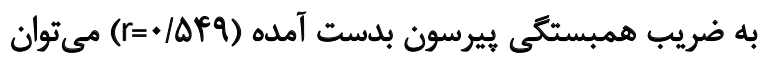
جنين استنباط كرد كه اين ارتباط مثبت است بحث

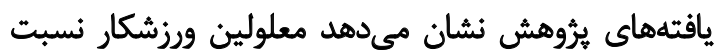

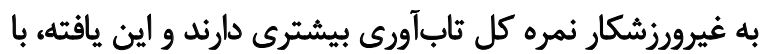

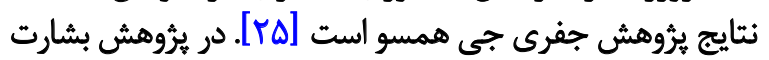

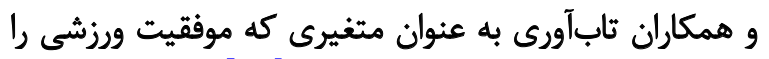

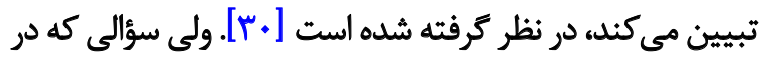




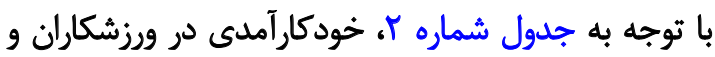

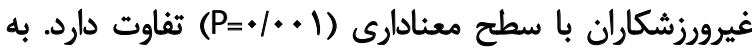

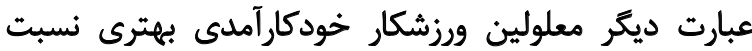

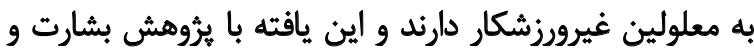

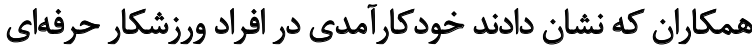

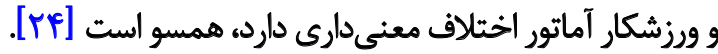

جكسون وهمكاران نيز در يثوهش خود نشان دادند ورزشكاران

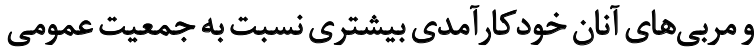

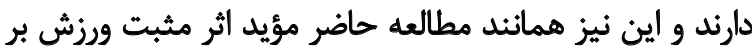

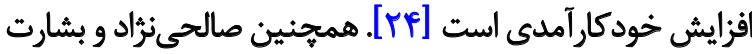

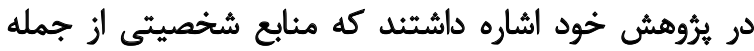

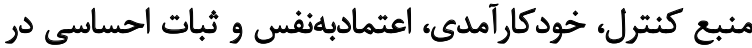

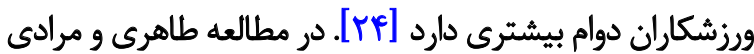

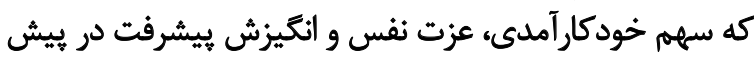

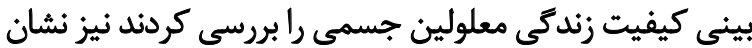

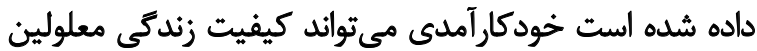

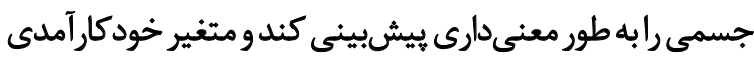

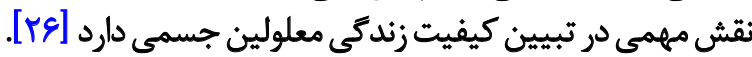

كشاورز و همكاران در بررسى مقايسهاى خودكارآمدى

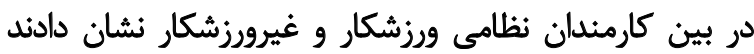

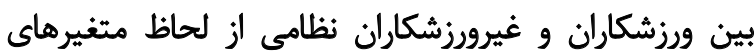

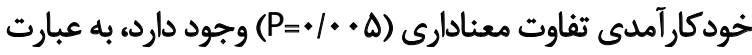

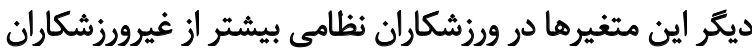

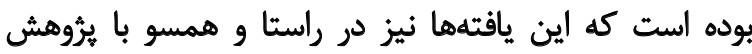

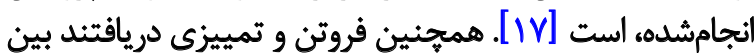

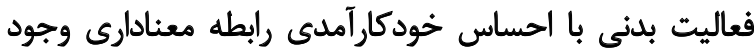

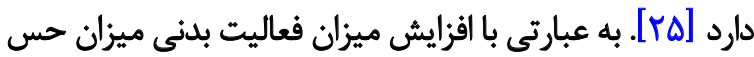

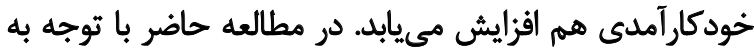

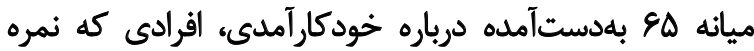

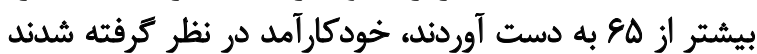

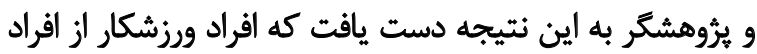

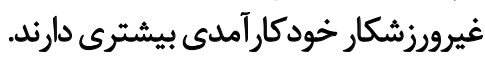

در تبيين اين يافته مىتوان كفت قدرت خودكارآمدى، به به بهار

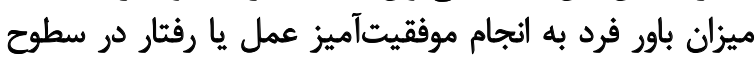

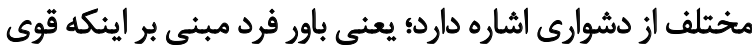

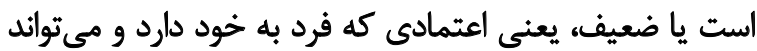

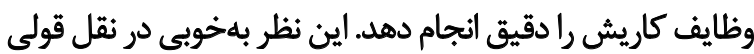

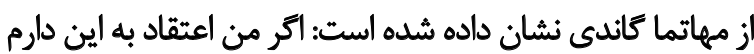

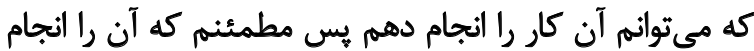

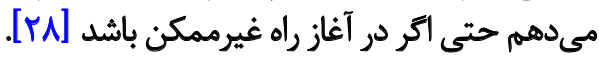

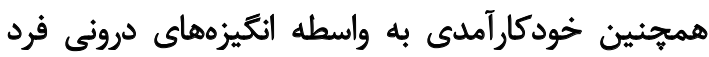

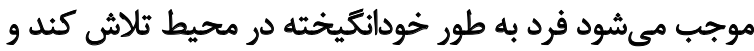

بيشينه) مى تواند با افزايش توان جسمى و فكرى به بهبود وضعيت

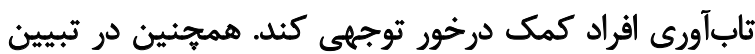

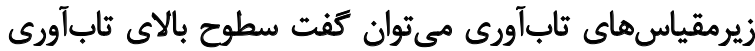

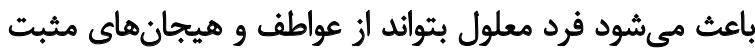

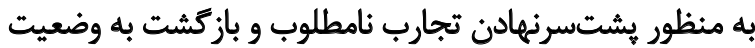

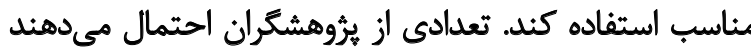

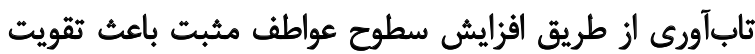

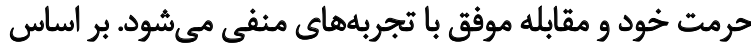

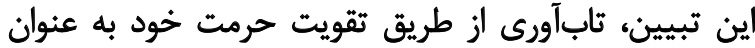

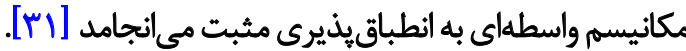

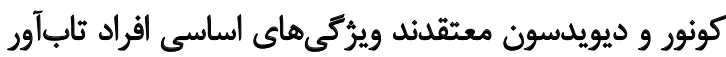

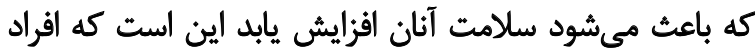

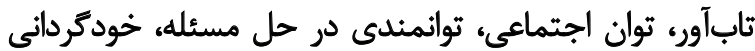

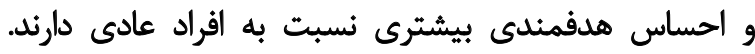

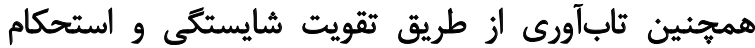

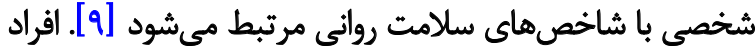

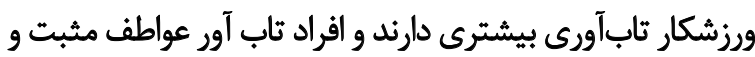

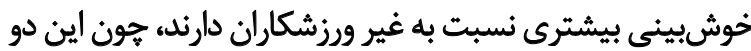

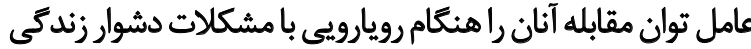

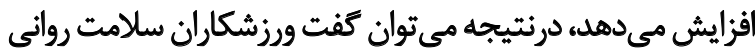

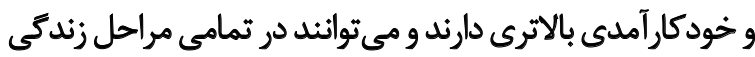

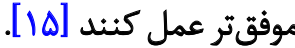

مهاركرى، يعنى توانايى فرد در مهار و مديريت شرايط كه يكى

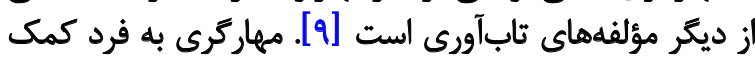

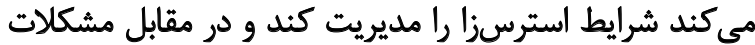

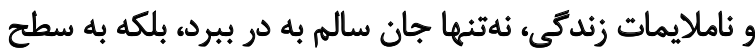

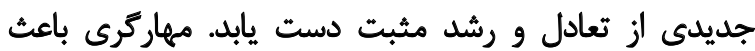

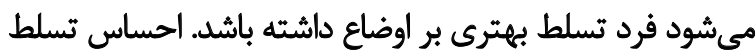

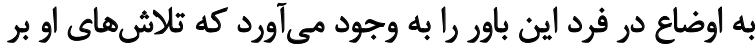

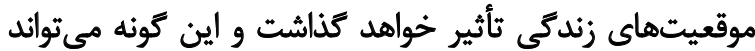

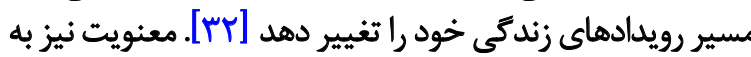

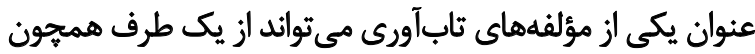

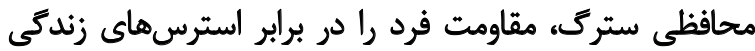

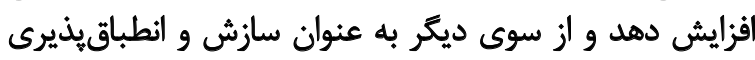
مثبت، فرد را به تعالى و كمال برساند [سبـ] نكتهاي كه در نتايج اين يُروهش حائز اهميت است، اينكه در

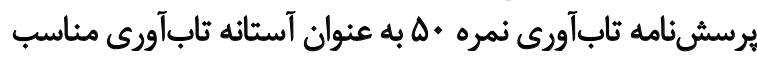

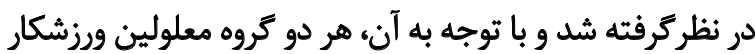

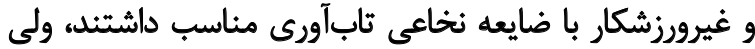

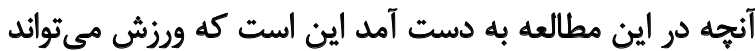

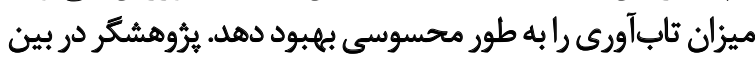

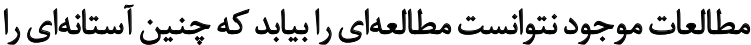
بين دو كروه ورزشكار و غيرورزشكار مقايسه كند. 
معتقدند هدف نهايى توانبخشى افراد آسيب نخاعى بها حداكثر

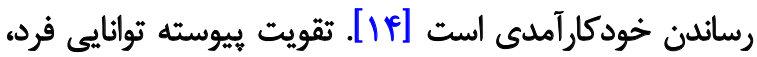

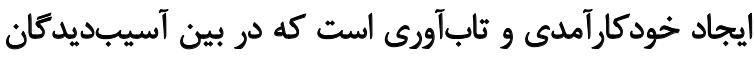

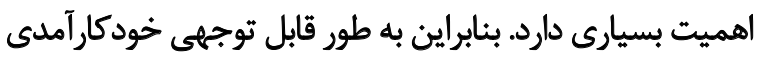

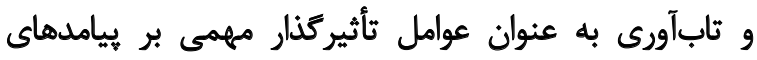
توانبخشى ناشى از آسيب نخاعى هستند.

\section{مفهوم سازي، ووش شناسي، نظارت، مرور و ويرايش}

با توجه به يافتههاى يزوهش حاضر و با درنظرترفتن نتايج

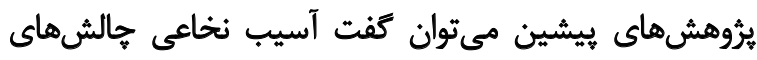

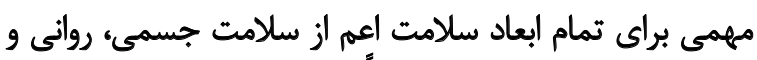

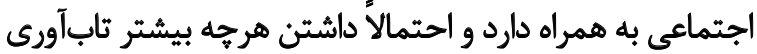

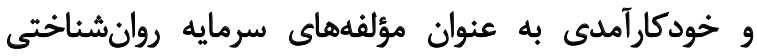

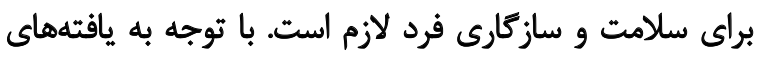

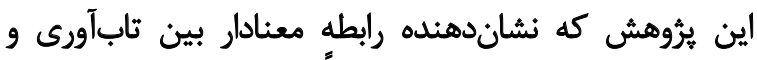

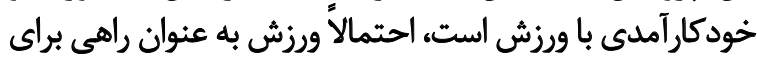
ارتقاى تاب آورى و خودكار آمدى در افراد بال با اضايعه نخاعى است.

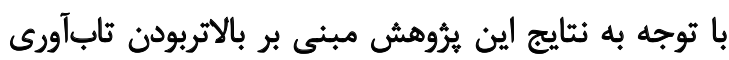

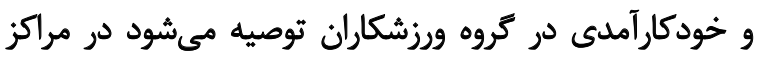

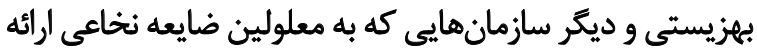

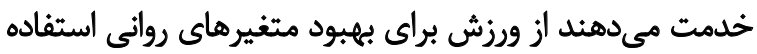

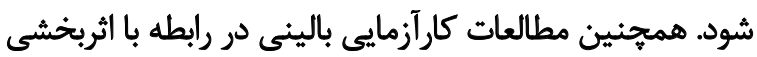

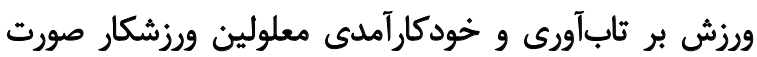

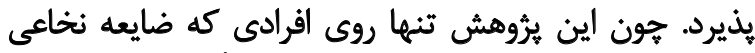

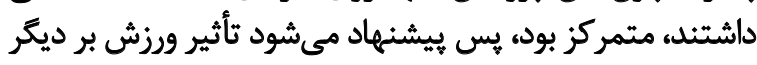

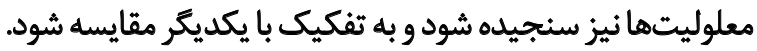

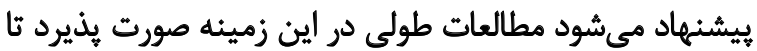

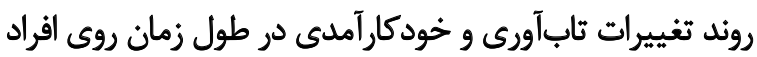

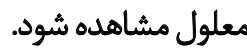

ملاحظات اخلاقى

\section{يبيروى أز اصول الخاق بوروهش}

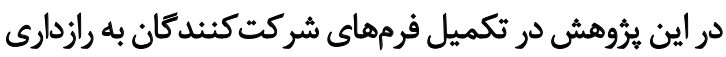

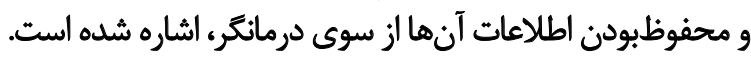

$$
\text { ماهي مالى }
$$

اين مقاله بركرفته از باياننامه كارشناسىارشد خانم نيلوفر

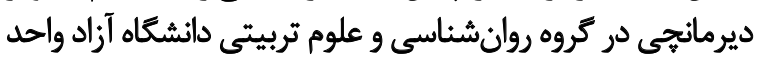

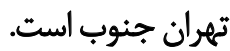
مشاركت نويسند كَان مفهومسازى، روششناسى، هرور و ويرايش: همه نويسندكان؛
تلاش هرجه بيشترى براى ادامه و تكميل تكاليف، عمل يا رفتار

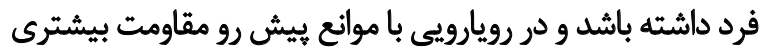

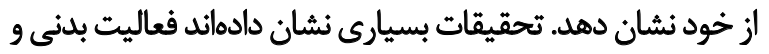

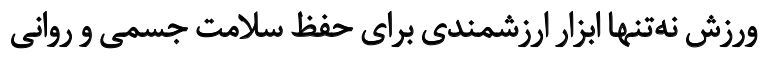

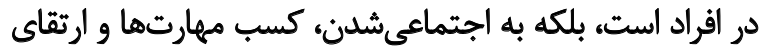
خودكار آمدى افراد كمك مي كند.

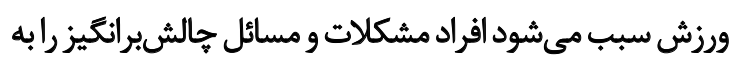

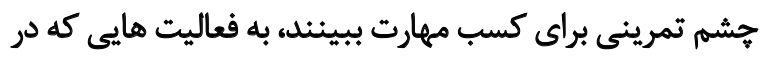

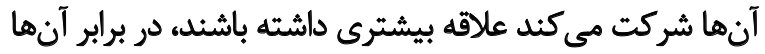

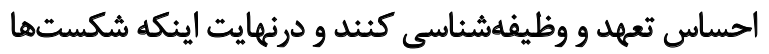

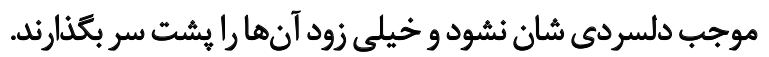

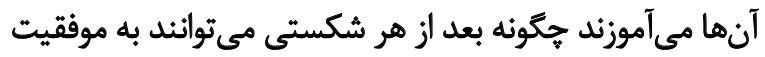

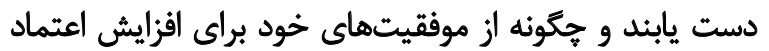

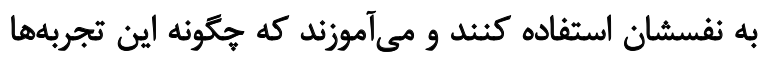

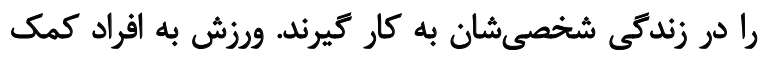

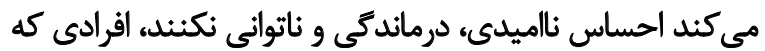

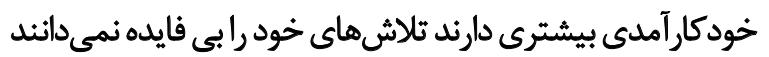

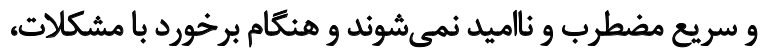

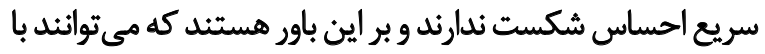

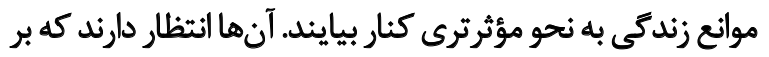

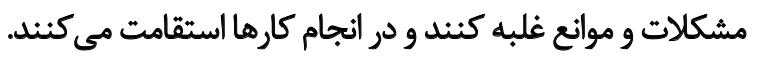
همجنين در تبيين رابطه بين تابآورى و خود كارآمدى بايد

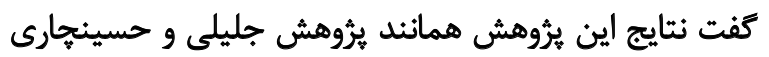

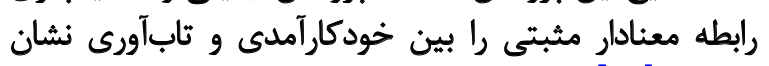

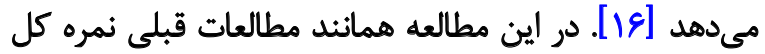

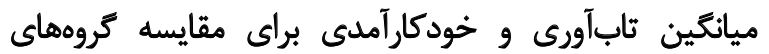

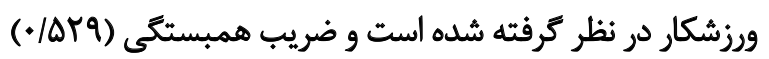

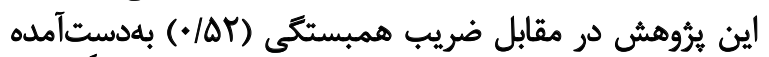

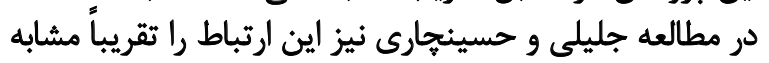

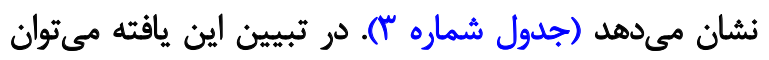

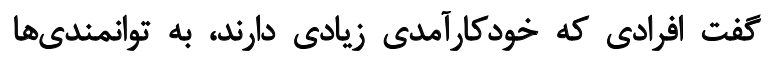

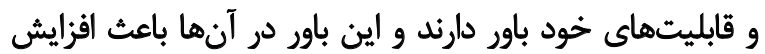

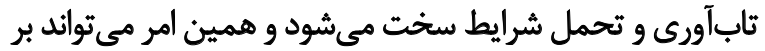
ديكَر ابعاد زندكى افراد با ضايعه نخاعى مؤثر واقع شود تثيجهنيرى

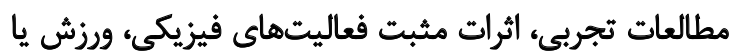

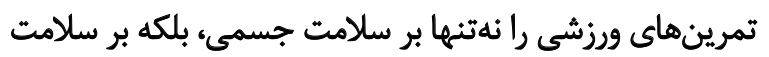

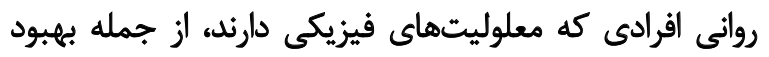
مديريت مشكلات خلقى، كاهش استرس، كاهش على علائم افسردئى

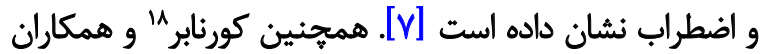


تحليل تحقيق، منابع، يردازش اطلاعات، نكارش و تهيه بِيشنويس

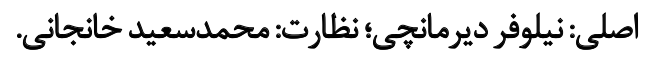

$$
\text { ت مارض مناقع }
$$

بنابر اظهارنظر نويسندكان، اين مقاله تعارض منافع ندارد.

$$
\text { تشكر و قدردانى }
$$

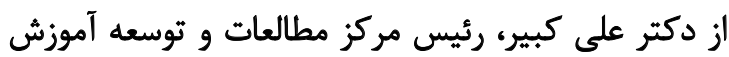

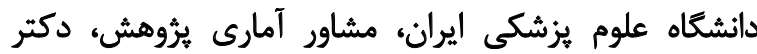

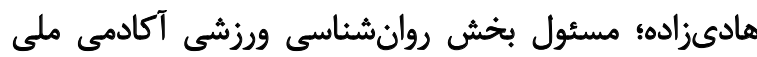

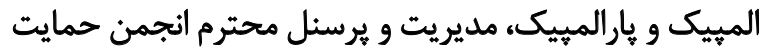

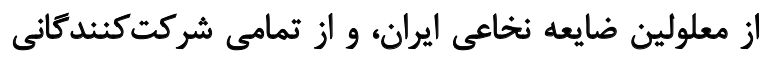
تشكر مى كنيهم. 


\section{References}

[1] World Health Organization. Summary: World report on disability. 2011. Geneva: World Health Organization; 2011.

[2] Mallet R, Runswick-Cole K. Approaching disability: Critical issues and perspectives. London: Routledge; 2014. [DOI:10.4324/9781315765464]

[3] Chulay M, Burns SM. Essentials of critical care nursing, $2^{\text {nd }}$ edition. American Association of Critical-Care Nurses. New York: McGraw Hill Medical; 2010.

[4] Karimi Darmani H. [Rehabilitation of specific groups with emphasis on social work (Persian)]. Tehran: Roshd; 2011.

[5] World Health Organization. Disability and rehabilitation: International perspectives on spinal cord injury. Geneva: World Health Organization; 2013.

[6] Nikbakht A, Bastami A, Norouzi Tabrizi K, Pashaei Sabet F, Gomarverdi S. Exploring home-based rehabilitation in traumatic spinal cord injury patients: A qualitative study. Iranian Journal of Nursing Research. 2017; 11(6):32-41. [DOI:10.21859/ijnr-11065]

[7] Machida M, Irwin B, Feltz D. Resilience in competitive athletes with spinal cord injury: The role of sport participation. Qualitative Health Research. 2013; 23(8):1054-65. [DOI:10.1177/1049732313493673] [PMID]

[8] Rahmani Rasa A, Hosseini SA, Haghgoo H, Khankeh HR, Ray G. [Review paper: Aspects related to resilience in people with spinal cord injury (Persian)]. Journal of Rehabilitation. 2017; 17(4):350-9. [DOI:10.21859/jrehab-1704350]

[9] Connor KM, Davidson J. Development of a new resilience scale: The Connor-Davidson Resilience Scale (CD-RISC). Depression and Anxiety. 2003; (18):76-82. [DOI:10.1002/da.10113] [PMID]

[10] Kordmirza Nikoozdade E. [Resilience: Concepts, theories, models, applications, including resonace-therapy protocols (Persian)]. Tehran: Abezh; 2013.

[11] Gillespe BM, Chaboyer W, Wallis M, Grimbeek P. Resilience in the operating room: Developing and testing of a resilience model. Journal of Advanced Nursing. 2007; 59(4):427-38. [DOI:10.1111/ j.1365-2648.2007.04340.x] [PMID]

[12] Soleymani E, Hoveyda R. [Investigating the concept of self-efficacy in Bandura's social cognition theory (Persian)]. Social Science Book of Month. 2013; 63:91-7.

[13] Bandura A, Schunk DH. Cultivating competence, self-efficacy, and intrinsic interest through proximal self-motivation. Journal of Personality and Social Psychology. 1981; 41(3):586-98. [DOI:o $\mathrm{rg} / 10.1037 / 0022-3514.41 .3 .586]$

[14] Kornhaber R, Mclean L, Betihavas V. Cleary M. Resilience and the rehabilitation of adult spinal cord injury survivors: A qualitative systematic review. Journal of Advanced Nursing. 2018; 74(1):23-33. [DOI:10.1111/jan.13396] [PMID]

[15] Ramazani F, Hejazi M. [A comparison of mental health, resilience and hardiness in a sample of athletes and non-athletes (Persian)]. Clinical Psychology and Personality. 2017; 15(1):157-66.

[16] Jalili A, Hosseinchari M. [Explaining psychological resilienc based on self-efficacy in athlete university student (Persian)]. Growth and Exercise-Sports Learning. 2010; 6:131-53.
[17] Keshavarz H, Shirvani H, Sobhani V, Barari A. [Comparison of resilience and self-efficacy among athletic and non-athletic military personnel (Persian)]. Journal of Police Medicine. 2016; $4(4): 227-34$

[18] Shokri B, Zareei M, Saharei O. [Comparing the Mental health of the athletes and non-athletes sensory-disabled people (Persian)]. Social Working. 2015; 4(3):37-43.

[19] Bakhshayesh H, Bahmani F, Kamali M. Comparative of mental health in disabled people of athletic and non-athletic. Iranian Journal of War and Public Health. 2012; 5(1):22-6.

[20] Hosseini SH, Kazemi SH, Shahbaznezhad L. [Evaluation of mental health in athletic and non athletic students (Persian)]. Journal of Mazandaran University of Medical Sciences. 2006; 15(53):97-104

[21] Mousavi Gilani S, Kianpour M, Sadeghi Khorashad M. [Comparison of mental health of athlete and non-athlete male students (Persian)]. Zahedan Journal of Research in Medical Sciences. 2002; 4(1):39-45.

[22] Gioia MC, Cerasa MA, di Lucente L, Brunelli S, Castellano V, Traballes M. Psychological impact of sports activity in spinal cord injury patients. Scandinavian Journal of Medicine \& Science in Sports. 2006; 16(6):412-6. [DOI:10.1111/j.1600-0838.2005.00518.x] [PMID]

[23] Martin JJ. Multidimensional self-efficacy and affect in wheelchair basketball players. Adapted Physical Activity Quarterly. 2008; 25(4):275-88. [DOI:10.1123/apaq.25.4.275] [PMID]

[24] Jackson B, Whipp PR, Chua KL, Dimmock JA, Hagger MS. Students' tripartite efficacy beliefs in high school physical education: Within- and cross-domain relations with motivational processes and leisure-time physical activity. Journal of Sport \& Exercise Psychology. 2013; 35(1):72-84. [DOI:10.1123/jsep.35.1.72] [PMID]

[25] Forootan F, Tamizi A. [Relation of participation in physical activity with self-efficacy of employees of the General Department of Sports and Youth of Sistan and Baluchistan Province (Persian)]. Paper presented at: The $2^{\text {nd }}$ National Conference on New Achievements in Physical Education and Exercise. 14-15 September 2016; Chahbahar, Iran.

[26] Taheri S, Moradi A, Pajouhesh S. [The contribution of self-efficacy, self-esteem and motivation to progress in predicting the quality of life of physical disabilities (Persian)]. Quarterly Psychology of Exceptional Individuals. 2013; 3(9):145-70.

[27] Khorram R. Jabari P. [The relationship between resiliency and mental health in scince education student of farhangian university-Shiraz (Persian)]. Paper presented at: Global Conference on New Horizons in Humanities, Future Studies and Empowerment. 28 January 2016; Shiraz, Iran.

[28] Narimani M, Abbasi M. [Investigating the relationship between psychological hardiness and self-reflection with burnout (Persian)]. Journal of Productivity Management. 2009; 2(8):75-92.

[29] Haghranjbar F, Kakavand A, Borjali V, Barmas H. Resilience and quality of mothers with mentally retarded children. Journal of Behavioral Sciences. 2011; 1(1):177-187.

[30] Besharat MA, Salehi M, SHahmohammadi KH, Nadali H, Zebardast A. [Relations of resilience and hardiness with sport achievement and menatal health in a sample of athletes (Persian)]. Contemporary Psychology. 2008; 3(2):38-49. 
[31] Carle AC, Chassin L. Resilience in a community sample of children of alcoholics: Its prevalence and relation internalizing symptomatology and positive affect. Journal of Applied Developmental Psychology. 2004; 25(5):577-95. [DOI:10.1016/j.appdev.2004.08.005]

[32] Richardson GE. The metatheory of resilience and resiliency. Journal of Clinical Psychology. 2002; 58(3):307-21. [DOI:10.1002/ jclp.10020

[33] Kobasa SC, Maddi SR, Kahn S. Hardiness and health: A prospective study. Journal of Personality and Social Psychology. 1982; 42(1):168-77. [DOI:10.1037/0022-3514.42.1.168] 
This Page Intentionally Left Blank 